\author{
ŁUKASZ PIĄTKOWSKI \\ Uniwersytet im. Adama Mickiewicza w Poznaniu \\ lukasz.piatkowski@amu.edu.pl \\ ORCID: 0000-0001-9092-6189
}

\title{
Zur korpusbasierten Vermittlung der Funktionsverbgefüge im DaF-Unterricht. Syntagmatische Muster als "Gebrauchsanleitung" für Deutschlernende
}

\author{
On corpus-based teaching of light verb \\ constructions in German during foreign \\ language lessons. Syntagmatic patterns \\ as "instructions for use" for learners of German
}

\begin{abstract}
The aim of the study is to show the possibilities of using corpus analyses for the introduction of light verb constructions in German during foreign language lessons. The corpus analyses of three selected light verb constructions Maßnahmen ergreifen, einen Beschluss fassen and Bericht erstatten show the co-occurrence of these units and enable the creation of syntagmatic patterns and the allocation of individual light verb constructions to thematic areas. The results of the analysis confirm the thesis that syntagmatic patterns are an aid to learners of German, as they present their use in a sentence and show the partner words with their syntactic function. This method can present the context of use and the thematic area of the light verb constructions. The results of the analyses are also a guideline to placing the syntagmatic patterns in German-Polish dictionaries to help learners to use them and communicate with them correctly.

KEYWORDS: light verb constructions, German-Polish dictionaries, The Mannheim German Reference Corpus (DeReKo), co-occurrence, syntagmatic pattern, language domain, German as a foreign language lesson.
\end{abstract}

SCHLÜSSELWORTE: Funktionsverbgefüge, deutsch-polnische Wörterbücher, Deutsches Referenzkorpus DeReKo, Kookkurrenz, syntagmatisches Muster, Sachgruppe, DaF-Unterricht. 


\section{EINLEITUNG}

Funktionsverbgefüge (nachstehend: FVG) stellen eine Herausforderung für Lernende des Deutschen als Fremdsprache dar. Als Mehrwortlexeme bereiten sie den Lernenden Schwierigkeiten beim Verstehen und beim Gebrauch in der Alltags- sowie Fachkommunikation. Das Ziel des vorliegenden Beitrags ist es zu zeigen, wie die Korpusarbeit bei der Vermittlung von FVG im DaF-Unterricht helfen und wie sie in den Sprachunterricht miteinbezogen werden kann. Es werden drei Fallstudien unternommen, die darauf abzielen, mithilfe von Kookkurrenzlisten syntagmatische Muster zu erstellen sowie die FVG den Sachgruppen zuzuordnen. Zusätzlich werden drei deutsch-polnische Wörterbücher darauf geprüft, ob sie die typischen Partnerwörter und Gebrauchsbeispiele beinhalten, wie die FVG in die Wörterbuchstruktur integriert werden, sowie ob die morpho-syntaktischen Informationen über Strukturtypen in den Wörterbuchartikeln vorhanden sind. Mittels der Ergebnisse lassen sich Implikationen für die Fremdsprachendidaktik sowie die Lexikographie erkennen. Syntagmatische Muster sind als ein Hinweis für Lernende anzusehen, wie die komplexen Strukturen des Deutschen (hier: FVG) gebraucht werden. Die Zuordnung zu Sachgruppen lässt schlussfolgern, in welchen Kontexten FVG verwendet werden können sowie in welchen Textsorten sie bevorzugt werden. Die Überprüfung der lexikographischen Werke erlaubt es, die Mikrostruktur der zweisprachigen Wörterbücher mit den Kookkurrenzen und Sachgruppen zu vervollständigen bzw. zu erweitern.

\section{FUNKTIONSVERBGEFÜGE IM DAF-UNTERRICHT}

Winhart (2005) schreibt,

eine klare, eindeutige Definition für Funktionsverbgefüge (FVG) ist in der Literatur nicht zu finden, obwohl der Begriff FVG gerade in der traditionellen Grammatik des Deutschen eine wichtige Rolle spielt. Aus den verschiedenen Definitionsansätzen lassen sich einige zentrale Kriterien extrahieren, die zur Eingrenzung der oft intuitiven Bestimmung einer Nomen-Verb-Kombination als FVG verwendet werden (Winhart 2005: 1).

Als FVG ${ }^{1}$ gelten in der germanistischen Sprachwissenschaft Verbindungen „eines weitgehend einer selbständigen lexikalischen Bedeutung entleerten

${ }^{1}$ Andere Bezeichnungen für FVG im Deutschen: Nominalstil, Substantivierungstendenzen, Streckformen des Verbs, Verbaufspaltungen, nominale Umschreibungen (vgl. Woźniak 2016: 132); im Polnischen: analityzmy werbo-nominalne, konstrukcje werbo-nominalne, predy- 
finiten Verbs mit einer Nominal- oder einer Präpositionalphase" (Busse 2002: 412) z.B. Angst machen oder zur Aufführung bringen. FVG bilden eine semantische Einheit und sind somit als voller Prädikatsausdruck anzusehen. Der Hauptbedeutungsträger dieser Kombinationen ist das Substantiv, dagegen übernimmt das Verb, das als Funktionsverb bezeichnet wird (vgl. Helbig \& Buscha 2013: 68), die Rolle des Stützelementes. Funktionsverben drücken hauptsächlich grammatische Eigenschaften wie Passivierung, aber auch semantische Merkmale wie Aktionsart aus. Funktionsverben können „einen Zustand [dur], eine Zustandsveränderung [incho] oder das Bewirken einer Zustandsänderung (bzw. eines Zustandes) [caus]“ (Helbig \& Buscha 2013: 70) bei dem Nomen markieren. Weitere Merkmale der FVG, z.B. Satzgliedschaft des nominalen Elementes, Paraphrasierbarkeit, Anaphorisierbarkeit, Erfragbarkeit, sowie Subklassifizierung der FVG werden z.B. bei Schmidt (1968), Helbig (1984), Polenz (1989), Kamber (2008), Hentschel und Weydt (2013) ausführlich dargestellt. In der einschlägigen Literatur werden

Funktionsverbgefüge [...] z.B. in Polenz (1987) als eine Untergruppe der Nominalisierungsverbgefüge aufgefasst, die eine kausative, inchoative, durative, aspektuale, passive "Eigenbedeutung" aufweisen, vgl. verbinden - in Verbindung treten. Nominalisierungsverbgefüge, die keine Funktionsverbgefüge sind, d. h. keine „Eigenbedeutung” haben, nennt Storrer (2006) Streckverbgefüge, z. B. Frage stellen - fragen, Unterricht erteilen - unterrichten (Taborek 2017: 2).

Taborek spricht das Problem der Äquivalenz der FVG an. FVG lassen sich durch ihnen zugrundeliegende Vollverben ohne Bedeutungsveränderung nicht ersetzen. FVG werden benutzt, um ein Geschehen bzw. die Tätigkeit genauer als das Vollverb $\mathrm{zu}$ beschreiben. Streckverbgefüge (im Weiteren: SVG) werden mit den entsprechenden Vollverben austauschbar benutzt, weil sie keine Bedeutungsdivergenzen aufweisen (vgl. Storrer 2013: 198).

Das Problem bei der Vermittlung der FVG im DaF-Unterricht und beim Gebrauch beruht darin, dass die Lernenden über die strukturellen und syntaktischen, beispielsweise Satzklammerbildung, sowie die semantischen Eigenschaften der FVG, wie Aktionsart, nicht informiert werden. Sie wissen nicht, dass FVG als eine semantisch zusammenhängende Einheit betrachtet werden und die jeweiligen Komponenten das volle Prädikat im Satz bilden. Ansonsten sind sie sich dessen nicht bewusst, wie FVG in die Struktur des Satzes integriert werden. Die häufigsten Ansätze zur Darstellung der FVG

\footnotetext{
kacja analityczna, wyrażenia opisowe, orzeczenia peryfrastyczne, im Englischen: support verb constructions, light verb constructions, verbo-nominal constructions, im Französischen: construction à verbes support (vgl. Domińczak 2014).
} 
im DaF-Unterricht beziehen sich auf das Auswendiglernen von Tabellen mit Äquivalenten in der Muttersprache. Werden die Lernergrammatiken untersucht, z.B. von Helbig und Buscha (1997), Hall und Scheiner (2001), Dreyer und Schmitt (2010), lässt sich schlussfolgern, dass sich die Übungen zu FVG nur auf Transformation der Sätze, Ersetzen der Verbformen und Wählen des richtigen Funktionsverbs konzentrieren. Außerdem werden die Sätze in Schulgrammatiken nicht situationsangemessen und nicht kontextabhängig gewählt. Dementsprechend scheint es begründet zu sein, neue methodologische sowie organisatorische Konzepte und Vorgehensweisen bei der Vermittlung der FVG im DaF-Unterricht zu erarbeiten, die dazu beitragen können, die Nomen-Verb-Verbindungen rezeptiv und produktiv zu beherrschen.

\section{DEUTSCHES REFERENZKORPUS}

Über den Einsatz von Korpora im Unterricht gibt es mehrere Publikationen, z.B. von Bernardini (2004), Lüdeling und Walter (2009), Siepmann (2009), Chrissou (2011), Ahrenholz und Wallner (2013) und Dziurewicz (2018). Das wachsende Interesse an Korpusarbeit begünstigt das Erlernen einer Fremdsprache, worauf Chrissou aufmerksam macht.

Die aktive Beschäftigung mit authentischem Sprachmaterial aus Korpora begünstigt das Erlernen sprachlicher Strukturen in ihrem kommunikativen Kontext. Dies hängt damit zusammen, dass Korpustexte in Bezug auf das Thema und Textsorte eine breite Streuung aufweisen und den realen Sprachgebrauch widerspiegeln (Chrissou 2011: 1).

Korpora erlauben es, unter anderem dank der Kookkurrenzlisten, also Listen mit den am häufigsten mit einer lexikalischen Einheit bzw. Wortgruppe auftretenden Partnerwörtern, den Anwendungskontext sowie Strukturtypen der jeweiligen Kombinationen aufzuzeigen.

Für den vorliegenden Beitrag werden das Deutsche Referenzkorpus DeReKo als die weltweit größte Sammlung von Texten und das Bearbeitungsprogramm COSMAS II ausgewählt. DeReKo ist eine „konzipierte Volltextdatenbank für das linguistisch motivierte Recherchieren in den Textsammlungen (= Korpora) des IDS2“ ${ }^{\prime \prime}$. Das DeReKo bilden über 42 Milliarden Wörter (nach Angaben der IDS Mannheim, Stand: 3.02.2018) der deutschen Sprache, die geschriebenen Texten aus der Gegenwart und neueren Vergan-

${ }^{2}$ http://www.ids-mannheim.de/cosmas2/projekt/einsteiger/was.html (Zugriff am: 5.08.2018). 
genheit entnommen wurden ${ }^{3}$. Die Einträge reichen bis in die 50er Jahre des 20. Jahrhunderts zurück. Die Verfügung über die Daten ist durch das Programm COSMAS II möglich, und zwar kostenlos. Die Sammlung besteht aus urheberrechtlich abgesicherten, vor allem belletristischen, wissenschaftlichen und populärwissenschaftlichen Texten. Im Korpus sind somit mehrere Textsorten zu finden, wie beispielsweise Protokolle, Zeitungstexte, öffentliche Reden von Politikern und andere.

Das Programm COSMAS II bietet unter anderem die Möglichkeit an, eine Kookkurrenzanalyse durchzuführen. Die Suche betrifft sowohl Wörter, Wortformen, Wortklassen als auch grammatische Strukturen, bei denen der Wort- und Satzabstand des Gesuchten bestimmt werden kann. Bei der Suche kann auf die Funktionswörter, die sog. Synsemantika, verzichtet werden. Die Ergebnisse werden in Form einer Tabelle mit der Vorkommensanzahl angegeben, was nicht nur für die quantitativen, sondern auch für die qualitativen Untersuchungen erforderlich ist, besonders beim Erstellen der Sprachgebrauchsmuster.

\section{FORSCHUNGSOBJEKT UND METHODOLOGIE}

Für die Zwecke des vorliegenden Beitrags werden die FVG Maßnahmen ergreifen, einen Beschluss fassen und Bericht erstatten in drei Schritten untersucht. Es wird gezeigt, wie die Korpusanalysen im DaF-Unterricht beim Erlernen der genannten Strukturen behilflich sein können. Die erste Etappe der Analyse bildet die Untersuchung der deutsch-polnischen Wörterbücher, und zwar PONS Duży stownik niemiecko-polski (2001) (im Weiteren: PONS 2001), PONS Großwörterbuch Deutsch-Polnisch (2007) (im Weiteren: PONS 2007) und PWN Großwörterbuch Deutsch-Polnisch (2010) (im Weiteren: PWN 2010). Es ist festzustellen, ob die Wörterbücher FVG als feste Bestandteile der Mikrostruktur verzeichnen und ob die FVG mit entsprechenden Gebrauchsbeispielen versehen werden. Im Weiteren werden die Korpusanalysen unternommen. Es werden Kookkurrenzlisten der jeweiligen Kombinationen erstellt, die es erlauben, die häufigsten Partnerwörter der FVG zu nennen und auf dieser Grundlage syntagmatische Muster zu bilden. Hunston und Francis (2000) verstehen den Begriff des syntagmatischen Musters wie folgt: unter Muster, oder pattern werden alle Wörter und Strukturen verstanden, die regelmäßig gemeinsam mit einem Wort oder einer Wortgruppe vorkommen. Das Pattern wird durch eine hohe Vorkommensfrequenz der Wörterkombination bestimmt (vgl. Mukherjee 2009: 106). Um die Umset-

\footnotetext{
${ }^{3}$ http://www1.ids-mannheim.de/kl/projekte/korpora.html (Zugriff am: 5.08.2018).
} 
zung der Muster darzustellen, werden Verwendungsbeispiele dem Korpus DeReKo entnommen. FVG werden in den Verwendungsbeispielen fettgedruckt und die jeweiligen syntaktischen Funktionen, die aufgrund der Konkordanzanalyse zugeordnet werden, kursiv markiert.

\section{FALLSTUDIE: MASSNAHMEN ERGREIFEN}

Das FVG Maßnahmen ergreifen wird in den analysierten Wörterbüchern wie folgt dargestellt.

Tabelle 1. FVG Maßnahmen ergreifen in den deutsch-polnischen Wörterbüchern

\begin{tabular}{|c|c|c|}
\hline Wörterbuch & Маßnаhme & ergreifen \\
\hline PONS 2001 & $\begin{array}{l}\sim \mathbf{n} \text { [gegen etw] ergreifen podejmować } \\
\text { [perf podjąć] kroki [przeciw czemuś] }\end{array}$ & $\begin{array}{l}\text { (in die Wege leiten) Maßnahmen przed- } \\
\text { sięwziąć kroki }\end{array}$ \\
\hline PONS 2007 & $\begin{array}{l}\sim \mathbf{n} \text { [gegen etw] ergreifen [o treffen] } \\
\text { podejmować [perf podjąć] kroki [prze- } \\
\text { ciw czemuś] }\end{array}$ & $\begin{array}{l}\text { Maßnahmen przedsięwziąć kroki; es } \\
\text { müssen dringend Sicherheitsmaß- } \\
\text { nahmen ergriffen werden natych- } \\
\text { miast należy podjąć kroki zmierzające } \\
\text { do zapewnienia bezpieczeństwa }\end{array}$ \\
\hline PWN 2010 & $\begin{array}{l}\text { bestimmte } \sim \text { n ergreifen a. vornehmen } \\
\text { podjąć określone działania }\end{array}$ & Maßnahmen podjąć działania a. kroki \\
\hline
\end{tabular}

Das FVG kommt in allen Wörterbüchern unter dem Lemma des Funktionsverbs sowie des Substantivs vor. Es ist anzumerken, dass es in PONS 2001 und PONS 2007 auf die präpositionale Rektion des FVG hingewiesen wird. Das FVG wird mit der Präposition gegen verwendet. Ansonsten fällt es auf, dass es im PONS 2007 unter dem Lemma ergreifen einen Beispielsatz gibt, und zwar in der Passivform. Es ist ein Hinweis für die Lernenden, dass das FVG in Passivkonstruktionen Anwendung findet. Zusätzlich sind die Restriktionen hinsichtlich des Numerus des Substantivs zu sehen, denn das Substantiv Maßnahme kommt in der Regel im Plural vor. In keinem Wörterbuch werden die Sachgruppen oder typischen Verbindungen mit anderen Wörtern verzeichnet, außerdem werden die Sprachregister nicht markiert. Die Wörterbücher liefern wenige Informationen bezüglich der Strukturtypen und der stilistischen Aspekte des analysierten FVG. Lediglich im PONS 2007 wird der Gebrauch der Passivkonstruktion angedeutet, was so verstanden werden kann, als ob das FVG Maßnahmen ergreifen nur im Passiv benutzt würde. Dementsprechend scheint es sich zu lohnen, die Korpusanalysen im 
DaF-Unterricht in Betracht zu ziehen und die Informationen im Wörterbuch $\mathrm{zu}$ revidieren.

Die Korpusanalyse fängt mit der Bildung der Suchanfrage an, mithilfe derer die wichtigsten Partnerwörter des FVG Maßnahmen ergreifen aufgelistet werden. Die Suchanfrage wird nach Taborek (2011: 74) gebildet und lautet: (Maßnahmen /+s0 \&ergreifen) OR (\&ergreifen /+s0 Maßnahmen) ${ }^{4}$. Die genannte Suchanfrage ermöglicht, alle Sätze mit dem Substantiv Maßnahmen sowohl vor als auch nach den konjugierten Formen des Verbs ergreifen zu finden. Im Korpus werden 29103 Treffer gefunden. Die häufigsten Kookkurrenzen des FVG Maßnahmen ergreifen werden in der nachstehenden Tabelle präsentiert.

Tabelle 2. Kookkurrenzen des FVG Maßnahmen ergreifen

\begin{tabular}{|r|l|c|c|}
\hline Nr. & \multicolumn{1}{|c|}{ Kookkurrenz } & Anzahl & Prozentanteil \\
\hline 1. & notwendige(n) & 1072 & 3,68 \\
\hline 2. & geeignete(n) & 849 & 2,92 \\
\hline 3. & Regierung & 682 & 2,34 \\
\hline 4. & entsprechende(n) & 642 & 2,21 \\
\hline 5. & Landesregierung & 614 & 2,11 \\
\hline 6. & Bundesregierung & 443 & 1,52 \\
\hline 7. & (zum) Schutz & 379 & 1,30 \\
\hline 8. & Senat & 181 & 0,62 \\
\hline 9. & (zur) Verbesserung & 181 & 0,62 \\
\hline 10. & sofort & 148 & 0,51 \\
\hline
\end{tabular}

Der Tabelle ist zu entnehmen, dass das Substantiv Maßnahmen attribuiert werden kann, und zwar durch Adjektive wie notwendige(n), geeignete(n) oder entsprechende( $n)$, je nach dem Kontext mit dem bestimmten oder unbestimmten Artikel. Ansonsten ist festzustellen, welche Subjekte das FVG Maßnahmen ergreifen am häufigsten fordern, nämlich Regierung (682 Treffer), Landesregierung (614), Bundesregierung (443) und Senat (181). Die anderen Substantive, Schutz und Verbesserung, treten in der Funktion des Präpositionalobjektes bzw. des nachgestellten Attributes ${ }^{5}$ auf. Anhand der Kookkurrenzanalyse lassen sich folgende Sprachgebrauchsmuster ermitteln:

${ }^{4}$ Es ist von Vorteil, wenn der Lehrer die Suchanfrage den Lernenden deduktiv präsentiert. Auf diese Art und Weise kann Zeit im Unterricht gespart werden.

${ }^{5}$ Die Diskussion über die Satzgliedschaft der nominalen Elemente, die ans Substantiv angeschlossen werden, ist umstritten. Mehr dazu in: Helbig (1984: 181) und Eisenberg (2006: 310ff.). 
Tabelle 3. Syntagmatisches Muster des FVG Maßnahmen ergreifen

\begin{tabular}{|l|l|l|l|}
\hline \multicolumn{1}{|c|}{ SUBJEKT } & \multicolumn{1}{|c|}{-} & \multicolumn{1}{c|}{ OBJEKT/ATTRIBUT } \\
\hline $\begin{array}{l}\text { Regierung } \\
\text { Landesregierung } \\
\text { Bundesregierung } \\
\text { Senat }\end{array}$ & ergreift & Maßnahmen & $\begin{array}{l}\text { zum Schutz ... } \\
\text { zur Verbesserung ... }\end{array}$ \\
\hline
\end{tabular}

Das ermittelte Muster wird im nachstehenden Satz realisiert:

Beim Stichwort Kraftwerksstandorte wird vonseiten der LINKEN und der SPD gefordert, dass die Landesregierung Maßnahmen zur Standorterhaltung der Kraftwerke im Saarland ergreift und hier insbesondere die Möglichkeiten einer Beteiligung des Landes und der kommunalen Gebietskörperschaften an den Kraftwerken prüft (Protokoll der Sitzung des Parlaments Landtag des Saarlandes am 18.05.2011).

Das obengenannte Muster kann durch Adverbiale ergänzt werden, was anhand von Beispielsätzen aus dem Korpus DeReKo gezeigt werden kann.

Tabelle 4. Syntagmatisches Muster des FVG Maßnahmen ergreifen mit adverbialen Angaben

\begin{tabular}{|l|c|l|l|l|}
\hline \multicolumn{1}{|c|}{ SUBJEKT } & - & MODALES ADVERBIAL & - & OBJEKT/ ATTRIBUT \\
\hline $\begin{array}{l}\text { Regierung } \\
\text { Landesregierung } \\
\text { Bundesregierung } \\
\text { Senat }\end{array}$ & ergreift & $\begin{array}{l}\text { sofort } \\
\text { rechtzeitig } \\
\text { endlich }\end{array}$ & Maßnahmen & $\begin{array}{l}\text { zum Schutz ... } \\
\text { zur Verbesserung ... }\end{array}$ \\
\hline
\end{tabular}

Die Umsetzung des Musters wird im folgenden Beispielsatz markiert.

Der Vorstand habe sofort nach seinem Amtsantritt im Jahre 1994 Maßnahmen zur Kostenentlastung im gesamten Konzern ergriffen (Salzburger Nachrichten, 21.12.1998).

Wenn die Konkordanzen im Korpus betrachtet werden, lässt sich schlussfolgern, dass das FVG mit einer Infinitivkonstruktion kombiniert wird. Die Analyse zeigt auch, dass das FVG in einen Passivsatz eingebettet werden kann. Ansonsten tauchen folgende Modalverben mit dem Funktionsverbgefüge auf: die größte Vorkommensanzahl gilt für müssen (1 386 Belege), können (525) und sollen (268). Das Verb wollen (83) spielt eine geringere Rolle. Hingegen gibt es in diesem Korpus keine Verbindung mit mögen und mit dürfen. Es ist zu betonen, dass die im Korpus gefundenen Treffer auch Mo- 
dalverbkonstruktionen im Passiv enthalten, was häufig der Fall bei dem analysierten FVG ist. Das syntagmatische Muster des Passivsatzes mit einem Modalverb kann zwei unterschiedliche Formen zeigen. Im Vorfeld kann das Pronomen es als Platzhalter stehen.

Tabelle 5. Syntagmatisches Muster des FVG Maßnahmen ergreifen im Passivsatz mit Modal$\operatorname{verb}(1)$

\begin{tabular}{|l|l|c|l|l|}
\hline PLATZHALTER & MODALVERB & - & OBJEKT / ATTRIBUT & \multicolumn{1}{|c|}{-} \\
\hline es & $\begin{array}{l}\text { müssen } \\
\text { können } \\
\text { sollen }\end{array}$ & Maßnahmen & $\begin{array}{l}\text { zum Schutz ... } \\
\text { zur Verbesserung ... }\end{array}$ & $\begin{array}{l}\text { ergriffen } \\
\text { werden }\end{array}$ \\
\hline
\end{tabular}

Das Nomenabstraktum kann ins Vorfeld verschoben werden, unter Beachtung, dass das Pronomen es getilgt wird:

Tabelle 6. Syntagmatisches Muster des FVG Maßnahmen ergreifen im Passivsatz mit Modalverb (2)

\begin{tabular}{|l|l|l|l|}
\hline \multicolumn{1}{|c|}{} & OBJEKT/ATTRIBUT & \multicolumn{1}{|c|}{ MODALVERB } & \multicolumn{1}{c|}{-} \\
\hline Maßnahmen & $\begin{array}{l}\text { zum Schutz ... } \\
\text { zur Verbesserung } \ldots\end{array}$ & $\begin{array}{l}\text { müssen } \\
\text { können } \\
\text { sollen }\end{array}$ & ergriffen werden \\
\hline
\end{tabular}

Das Muster ist im nachfolgenden Beispielsatz zu sehen.

Außerdem sollen der Kampf gegen die Finanzierung des internationalen Terrorismus verstärkt und Maßnahmen zur Erhöhung der Flugsicherheit in der Europäischen Union ergriffen werden (Protokoll der Sitzung des Parlaments Deutscher Bundestag am 18.10.2001).

Anhand der Kookkurrenzliste und der Partnerwörter lässt sich das FVG Maßnahmen ergreifen den Sachgruppen nach Dornseiff (2004: 202) zuordnen. Das Miteinandervorkommen des FVG und der gefundenen Kookkurrenzen weisen darauf hin, dass das FVG im Bereich Gesellschaft, Recht und Wirtschaft und Finanzen verwendet wird6, was die Verwendungsbeispiele auch bestätigen.

\footnotetext{
${ }^{6}$ Es ist zu beachten, dass die Zuordnung zu Sachgruppen nicht völlig objektiv verläuft.
} 


\section{FALLSTUDIE: EINEN BESCHLUSS FASSEN}

Das FVG einen Beschluss fassen wird in die untersuchten Wörterbücher folgendermaßen eingetragen.

Tabelle 7. FVG Beschluss fassen in den deutsch-polnischen Wörterbüchern

\begin{tabular}{|l|c|c|}
\hline \multicolumn{1}{|c|}{ Wörterbuch } & Beschluss & fassen \\
\hline PONS 2001 & einen $\sim$ fassen podjąć decyzję & - \\
\hline PONS 2007 & einen $\sim$ fassen podjąć decyzję & - \\
\hline PWN 2010 & einen $\sim$ fassen podjąć decyzję & - \\
\hline
\end{tabular}

Das FVG einen Beschluss fassen kommt in den analysierten lexikographischen Werken unter dem Lemma Beschluss vor. In keinem der untersuchten Wörterbücher wird das FVG unter dem Lemma fassen notiert, obwohl das Verb ein Funktionsverb und somit ein fester Bestandteil des FVG ist. Es ist $\mathrm{zu}$ beachten, dass die eingetragenen Wörterbuchartikel nur die polnischen Äquivalente angeben und keine grammatischen bzw. morpho-syntaktischen Informationen aufweisen. Für Deutschlernende scheint es problematisch zu sein, denn sie bekommen keine Hinweise, wie das Mehrwortlexem im Satz gebraucht wird. Dementsprechend können die Lehrer und die Lernenden im Korpus DeReKo Auskunft über Valenz, Rektion und Satztypen, in denen das FVG benutzt wird, einholen.

Die Suchanfrage (Beschluss /+s0 \&fassen) OR (\&fassen /+s0 Beschluss) ergibt 25753 Treffer. Die unten beigefügte Tabelle beinhaltet die am meisten vorkommenden Partnerwörter des FVG.

Tabelle 8. Kookkurrenzen des FVG einen Beschluss fassen

\begin{tabular}{|r|l|c|c|}
\hline Nr. & \multicolumn{1}{|c|}{ Kookkurrenz } & Anzahl & Prozentanteil \\
\hline 1. & entsprechende(n) & 2312 & 8,98 \\
\hline 2. & einstimmig & 1444 & 5,61 \\
\hline 3. & Sitzung & 1244 & 4,83 \\
\hline 4. & Gemeinderat & 884 & 3,43 \\
\hline 5. & Stadtrat & 499 & 1,94 \\
\hline 6. & Landtag & 434 & 1,69 \\
\hline 7. & Lesung & 430 & 1,67 \\
\hline 8. & bereits & 245 & 0,95 \\
\hline 9. & Parlament & 187 & 0,73 \\
\hline 10. & Gemeindevertretung & 155 & 0,60 \\
\hline
\end{tabular}


Es ergibt sich aus der Tabelle, welche Substantive die Subjektfunktion in Sätzen mit dem FVG einen Beschluss fassen erfüllen, und zwar sind das Gemeinderat (884 Belege), Stadtrat (499), Landtag (434), Parlament (187) und Gemeindevertretung (155). Als Modaladverbien kommen einstimmig (1444 Belege) und bereits (245) vor. Die Tabelle liefert auch Informationen über die lokalen Angaben, die am häufigsten mit der Wortgruppe einen Beschluss fassen verbunden werden und die erst nach der Analyse der Konkordanzen zum Vorschein kommen, nämlich in der Sitzung (1244 Belege) und in der Lesung (430). Die Recherche zeigt, dass diese Konstruktion im Passiv vorkommen kann, und zwar als Vergangenheitsformen mit den Hilfsverben wurde (2929 Belege) sowie ist worden (734). Die Modalverben, die im Korpus erscheinen, sind sollen, das $636 \mathrm{Mal}$ vorkommt, und müssen mit 38 Belegen. Die anderen Modalverben werden nicht aufgeführt, was jedoch kein Beweis dafür ist, dass dieses FVG mit diesen nicht vorkommen kann. Anhand der Kookkurrenzanalyse lassen sich folgende Sprachgebrauchsmuster erstellen:

Tabelle 9. Syntagmatisches Muster des FVG einen Beschluss fassen

\begin{tabular}{|l|c|c|c|}
\hline \multicolumn{1}{|c|}{ SUBJEKT } & - & ATTRIBUT & - \\
\hline $\begin{array}{l}\text { Gemeinderat } \\
\text { Stadtrat } \\
\text { Landtag }\end{array}$ & fasst & entsprechenden & Beschluss \\
\hline
\end{tabular}

Das oben definierte Muster wird im folgenden Beispielsatz realisiert:

Der Gemeinderat Münchwilen hat einen grundsätzlichen Beschluss bezüglich der Auflage und der Finanzierung der in Entstehung begriffenen Gemeindechronik «50 Jahre Gemeinde Münchwilen» gefasst (St. Galler Tagblatt, 8.10.1997).

Die häufigsten attributiv gebrauchten Adjektive sind entsprechende(n) mit 2312 Belegen, endgültige(n) (109), gemeinsame(n) (89), gefolgt von folgende(n) (48). Die Zahlen beziehen sich nicht nur auf die angegebene Form des Adjektivs, sondern auch auf alle deklinierbaren Formen, die im DeReKo gefunden werden. Die Gestalt des oben genannten Musters lässt sich durch Adverbiale ergänzen.

Tabelle 10. Syntagmatisches Muster des FVG einen Beschluss fassen mit adverbialen Angaben

\begin{tabular}{|l|l|l|l|c|}
\hline \multicolumn{1}{|c|}{ SUBJEKT } & - & MODALES ADVERBIAL & LOKALES ADVERBIAL & - \\
\hline $\begin{array}{l}\text { Gemeinderat } \\
\begin{array}{l}\text { Stadtrat } \\
\text { Landtag }\end{array}\end{array}$ & fasst & $\begin{array}{l}\text { einstimmig } \\
\text { bereits }\end{array}$ & $\begin{array}{l}\text { in der Sitzung } \\
\text { in der Lesung }\end{array}$ & Beschluss \\
\hline
\end{tabular}


Das erweiterte Muster findet im folgenden Beispielsatz Anwendung:

Dazu fassten die Abgeordneten bereits in der Dezember-Sitzung einen Beschluss.

(Nordkurier, 21.03.2001)

Das FVG kommt vor allem im institutionellen Kontext vor. Gemäß der Analyse und der angeführten Beispiele lässt sich die Wortgruppe zu den Sprachbereichen bzw. zu den Sachgruppen Recht, Wirtschaft, Gesellschaft, menschliches Zusammenleben, Finanzen, Sport und Freizeit einordnen.

\section{FALLSTUDIE: BERICHT ERSTATTEN}

In den anvisierten Wörterbüchern wird das FVG in folgender Form verzeichnet.

Tabelle 11. FVG Bericht erstatten in den deutsch-polnischen Wörterbüchern

\begin{tabular}{|l|c|l|}
\hline Wörterbuch & \multicolumn{1}{|c|}{ Bericht } & \multicolumn{1}{|c|}{ erstatten } \\
\hline PONS 2001 & - & $\begin{array}{l}\text { form (mitteilen) [jdm] Bericht über etw } \\
(a k k) \sim \text { zdać [komuś] raport z czegoś }\end{array}$ \\
\hline PONS 2007 & $\begin{array}{l}\text { jdm über etw erstatten zdawać [perf } \\
\text { zdać] komuś z czegoś sprawozdanie }\end{array}$ & $\begin{array}{l}\text { form (mitteilen) [jdm] Bericht über etw } \\
(a k k) \sim \text { zdawać [perf zdać] [komuś] } \\
\text { raport z czegoś }\end{array}$ \\
\hline PWN 2010 & $\begin{array}{l}\text { einen über etw. + A. erstatten a. a. } \\
\text { geben złożyć raport z czegoś, zdać } \\
\text { relację z czegoś }\end{array}$ & $\begin{array}{l}\text { jmdm Bericht über etw. + A. } \sim \text { zdać } \\
\text { komuś relację z czegoś }\end{array}$ \\
\hline
\end{tabular}

In den analysierten Wörterbüchern sind die Informationen über die Valenz und Rektion des FVG vorhanden. An das Substantiv im FVG Bericht erstatten können Präpositionalphrasen angeschlossen werden, und zwar diejenigen mit der Präposition über. In jedem Wörterbuch wird das FVG unter dem Verb erstatten lemmatisiert, was nicht der Fall bei Bericht ist. Im Wörterbuch PONS 2001 kommt das FVG unter dem Substantiv nicht vor.

Für die Verbindung Bericht erstatten werden im Korpus 8922 Belege dokumentiert. Der häufigste Kookkurrent für Bericht erstatten ist regelmäßig (371 Belege) und gilt syntaktisch als Adverbialbestimmung. Die zwei nächsten Kookkurrenzen sind die Substantive Landtag (255) und Parlament (141), die syntaktisch als Dativobjekte gelten. Die Funktion des Dativobjektes erfüllen auch Ausschuss (135), Sicherheitsrat (85) und Rat (81). Als Subjekte treten Stadtrat (171) und Regierung (106) auf. 
Tabelle 12. Kookkurrenzen des FVG Bericht erstatten

\begin{tabular}{|c|l|c|c|}
\hline Nr. & \multicolumn{1}{|c|}{ Kookkurrenz } & Anzahl & Prozentanteil \\
\hline 1. & regelmäßig & 371 & 4,16 \\
\hline 2. & Landtag & 255 & 2,86 \\
\hline 3. & Parlament & 141 & 1,58 \\
\hline 4. & jährlich & 172 & 1,93 \\
\hline 5. & Stadtrat & 171 & 1,92 \\
\hline 6. & Ausschuss & 135 & 1,51 \\
\hline 7. & über die Lage & 131 & 1,47 \\
\hline 8. & Regierung & 106 & 1,19 \\
\hline 9. & Sicherheitsrat & 85 & 0,95 \\
\hline 10. & Rat & 81 & 0,91 \\
\hline
\end{tabular}

In Tabelle 12. gibt es eine Präpositionalphrase über die Lage mit 131 Belegen. Andere Präpositionalphrasen sind beispielsweise über den Stand (82 Belege), über die Aktivitäten (71), über die Tätigkeiten (48) und über die Umsetzung (44). Das Sprachgebrauchsmuster hat folgende Gestalt:

Tabelle 13. Syntagmatisches Muster des FVG Bericht erstatten

\begin{tabular}{|l|c|l|c|l|}
\hline \multicolumn{1}{|c|}{ SUBJEKT } & - & \multicolumn{1}{|c|}{ DATIVOBJEKT } & - & OBJEKT/ATTRIBUT \\
\hline $\begin{array}{l}\text { Stadtrat } \\
\text { Regierung }\end{array}$ & erstattet & $\begin{array}{l}\text { dem Landtag } \\
\text { dem Sicherheitsrat } \\
\text { dem Rat }\end{array}$ & Bericht & $\begin{array}{l}\text { über die Lage } \\
\text { über den Stand } \\
\text { über die Aktivitäten }\end{array}$ \\
\hline
\end{tabular}

Das oben genannte Muster wird mit allen seinen Slots im nachstehenden Beispielsatz realisiert:

Die Regierung erstattet dem Landrat Bericht über den Stand der Vorkehrungen beim Berufsvorbereitungsjahr (Die Südostschweiz, 10.04.2006).

Das Substantiv im FVG kann durch Adjektive attribuiert werden. Im Korpus DeReKo haben die Adjektive ausführlichen (47) und zuständigen (42) die höchste Vorkommensanzahl. Das FVG kommt auch mit modalen Adverbialbestimmungen vor, von denen regelmäßig (371 Belege), jährlich (172), ausführlich (85), halbjährlich (27) und detailliert (27) am häufigsten belegt werden. Die Modifikation des Gebrauchsmusters durch die Modalverben sollen (306) und müssen (235) ist möglich, was im folgenden Beispielsatz realisiert wird: 
Seinem Plan zufolge sollen die Mitgliedstaaten der Kommission über die Testergebnisse regelmäßig Bericht erstatten (Luxemburger Tageblatt, 16.02.2013).

Das FVG Bericht erstatten wird zudem in Infinitivkonstruktion eingebettet. Verben, die eine Infinitivkonstruktion verlangen, in der Bericht erstatten als Prädikat vorkommt, sind unter anderem auffordern (36), verpflichten (31 Belege), und bitten (22). Das Sprachgebrauchsmuster mit Infinitivkonstruktion sieht folgendermaßen aus.

Tabelle 14. Syntagmatisches Muster des FVG Bericht erstatten

\begin{tabular}{|c|l|l|c|l|c|}
\hline SUBJEKT & \multicolumn{2}{|l|}{ KOMPLEXES PRÄDIKAT } & - & OBJEKT/ATTRIBUT & - \\
\hline $\begin{array}{l}\text { Stadtrat } \\
\text { Regierung }\end{array}$ & $\begin{array}{l}\text { ist } \\
\text { wird }\end{array}$ & $\begin{array}{l}\text { lufgefordert, } \\
\text { verpflichtet, } \\
\text { gebeten, }\end{array}$ & Bericht & $\begin{array}{l}\text { über die Lage } \\
\text { über den Stand }\end{array}$ & zu erstatten \\
\hline
\end{tabular}

Das gebildete Muster wird im folgenden Satz umgesetzt:

Vorstand und Aufsichtsrat sind verpflichtet, Bericht über die Umstände der Trennung von Dietmar Beiersdorfer zu erstatten (Hamburger Morgenpost, 26.06.2009).

Die oben präsentierten Sprachgebrauchsmuster sind ein Beweis dafür, dass das FVG in unterschiedlichen Konstruktionen modifiziert und mit zahlreichen sprachlichen Mitteln kombiniert werden kann. Aus der Analyse der Partnerwörter folgt, dass Bericht erstatten in den Sprachbereichen Wissenschaft, menschliches Zusammenleben, Finanzen und Wirtschaft Anwendung findet.

\section{SCHLUSSBEMERKUNGEN UND DISKUSSION}

In dem vorliegenden Beitrag wurden drei FVG korpusbasiert analysiert. Die Analyse hat sich auf das linguistische Korpus DeReKo gestützt und hatte zum Ziel, die korpusbasierte Methode der Vermittlung von FVG im DaF-Unterricht vorzuschlagen. Aufgrund der Kookkurrenzanalyse wurden syntagmatische Muster der jeweiligen Mehrwortlexeme erstellt. Ansonsten haben die Kookkurrenzlisten dazu beigetragen, dass FVG den möglichen Sachgruppen zugeordnet wurden.

Die Analysen weisen mehrere Implikationen für den Fremdsprachenunterricht auf: die dargestellte Methode ermöglicht den Lehrenden die schnelle und erfolgsreiche Vermittlung der FVG im Unterricht. Mithilfe der Kook- 
kurrenzanalysen lassen sich die Komplementierung sowie Erweiterbarkeit der jeweiligen Konstruktionen definieren, was von Vorteil für die Lernenden zu sein scheint. Dank der Partnerwörterliste sind sich die Lernenden bewusst, in welcher Umgebung das FVG vorkommt und zusätzlich in welchen Sprachbereichen es Anwendung findet. Die Ergebnisse der Analyse präsentieren sich folgendermaßen:

- bei Bericht erstatten werden Dativobjekte obligatorisch benutzt,

- die modalen Angaben können als Erweiterung der erstellten Muster fungieren,

- Präpositionalphrasen mit lokativer Funktion sind auch bei manchen Konstruktionen obligatorisch, z.B. einen Beschluss fassen,

- es kommt vor, dass das Substantiv attribuiert wird, obwohl die Attribuierung bei den FVG in der Regel erschwert ist, z.B. entsprechende Maßnahmen ergreifen, einen Bericht über etwas erstatten.

Die metalexikographische Analyse hat gezeigt, dass nicht in allen Fällen das FVG im Wörterbuch verzeichnet wird. Es ist davon auszugehen, dass die Wörterbücher die Informationen über typische und häufige Verbindungen der jeweiligen Wörter und sogar Gebrauchsbeispiele beinhalten sollen. Nur im Falle von Maßnahmen ergreifen in PONS 2007 ist ein Passivbeispielsatz zu finden, bei den anderen untersuchten FVG werden Verwendungsbeispiele nicht präsentiert.

Die Studie hat nicht nur einen kontrastiven und didaktischen, sondern auch lexikographischen, lexiko-grammatischen, terminologischen Charakter. Es wird darauf hingewiesen, in welcher Umgebung, bzw. in welcher Konstruktion FVG im Deutschen zum Vorschein kommen. Die Ergebnisse der Untersuchung können ein Impuls für die Korrektur, Erweiterung, und Ergänzung der deutsch-polnischen Wörterbücher sein, sowohl der Universal- als auch der Fachwörterbücher, denn laut Storrer gelten „FVG als typisch für amtssprachliche Texte [...], [und] werden [...] bisweilen genutzt, wenn ein Eindruck von Offizialität hervorgerufen werden soll“" (Storrer 2013: 176). Die Studie ist ein Anstoß für das Verfassen der Wörterbücher mit Einträgen zu FVG und deren Anwendung in konkreten Sprach- und Fachbereichen, z.B. Recht, Politik, Sport, Verwaltung.

Abschließend zeigt die Studie das Potenzial der Arbeit mit einsprachigen Korpora, die im Fremdsprachenunterricht Anwendung finden sollte. Die Korpusrecherche ermöglicht den Lernenden, Auskunft über die typischen Verbindungen der jeweiligen Wortkombinationen, den Sprachbereich und die Verwendung in konkreten Situationen einzuholen und sie mit Zahlenangaben zu belegen. Dementsprechend sollten die Fremdsprachenlehrer grammatische Strukturen und Wortverbindungen in Anlehnung an Kor- 
pusdaten ermitteln, indem sie die Beispielsätze dem Korpus entnehmen, in dem Texte mit lebendiger Sprache erfasst wurden.

Zum Schluss werden die erstellten syntagmatischen Muster mit den Variablen X, Y und Z (vgl. Mel'čuk 2001: 275) zusammengestellt, wobei X für Subjekte, $Y$ und $Z$ für andere Satzglieder steht.

Maßnahmen

$X$ ergreift Maßnahmen zum Schutz von $Y$

$X$ ergreift rechtzeitig Maßnahmen zum Schutz von $Y$

es müssen Maßnahmen zum Schutz von $Y$ ergriffen werden

Maßnahmen zum Schutz von $Y$ müssen ergriffen werden

\section{Beschluss}

\section{$X$ fasst einen Y-en Beschluss}

$X$ fasst einstimmig in der Sitzung am $Y$ einen Beschluss

Bericht

$X$ erstattet dem Y einen Bericht über Z

$X$ ist verpflichtet, einen Bericht über $Z$ zu erstatten

\section{LITERATURVERZEICHNIS}

Referenzliteratur

Ahrenholz, B. / Wallner, F. (2013). Digitale Korpora und Deutsch als Fremdsprache. In: B. Ahrenholz / I. Oomen-Welke (Hrsg.), Deutschunterricht in Theorie und Praxis. Deutsch als Fremdsprache (S. 261-272). Hohengehren: Schneider Verlag.

Bernardini, S. (2004). Corpora in the classroom. An overview and some reflections on future developments. In: J. Sinclair (Hrsg.), How to use corpora in language teaching (S. 15-36). Amsterdam-Philadelphia: John Benjamins Publishing Co.

Busse, D. (2002). Wortkombinationen. In: D. A. Cruse / F. Hundsnurscher / M. Job / P. R. Lutzeier (Hrsg.), Lexikologie. Ein internationales Handbuch zur Natur und Struktur von Wörtern und Wortschätzen. Handbücher zur Sprach- und Kommunikationswissenschaft (S. 408-415). Berlin-New York: de Gruyter.

Chrissou, M. (2011). Mit Textkorpora im Unterricht arbeiten und Sprachförderung initiieren. https://www.uni-due.de/imperia/md/content/prodaz/chrissou_korpusarbeit.pdf (Zugriff am: 5.08.2018).

Domińczak, A. (2014). Definicje analitycznych konstrukcji werbo-nominalnych. Ujęcie kontrastywne niemiecko-polskie. In: A. Stolarczyk-Gembiak / M. Woźnicka (Hrsg.), Zbliżenia: językoznawstwo - literaturoznawstwo - translatologia (S. 25-32). Konin: Państwowa Wyższa Szkoła Zawodowa w Koninie.

Dornseiff, F. (2004). Der deutsche Wortschatz nach Sachgruppen. Mit einer lexikographisch-historischen Einführung und einer ausführlichen Bibliographie zur Lexikographie und Onomasiologie. Berlin: de Gruyter. 
Dreyer, H. / Schmitt, R. (2010). Lehr- und Übungsbuch der deutschen Grammatik - aktuell. Warszawa: Hueber Polska.

Dziurewicz, E. (2018). Einsatz von Korpora als ein neuer Weg in der Phraseologieforschung Phraseografie, Phraseodidaktik, Phraseopragmatik, kontrastive Phraseologie. Studien zur Deutschkunde, Bd. LXI, 983-997.

Eisenberg, P. (2006). Grundriss der deutschen Grammatik. Band 2: Der Satz. Stuttgart-Weimar: J. B. Metzler.

Hall, K. / Scheiner, B. (2001). Übungsgrammatik. Deutsch als Fremdsprache für Fortgeschrittene. Ismaning: Max Hueber Verlag.

Helbig, G. (1984). Studien zur deutschen Syntax. Leipzig: VEB Verlag Enzyklopädie.

Helbig, G. / Buscha, J. (1997). Übungsgrammatik Deutsch. Leipzig-Berlin-München u.a.: Langenscheidt Verlag Enzyklopädie.

Helbig, G. / Buscha, J. (2013). Deutsche Grammatik. Ein Handbuch für den Ausländerunterricht. München: Klett-Langenscheidt.

Hentschel, E. / Weydt, H. (2013). Handbuch der deutschen Grammatik. Berlin-Boston: de Gruyter.

Hunston, S. / Francis, G. (2000). Pattern grammar: A corpus-driven approach to the lexical grammar of English. Amsterdam: John Benjamins.

Kamber, A. (2008). Funktionsverbgefüge - empirisch. Eine korpusbasierte Untersuchung zu den nominalen Prädikaten des Deutschen. Tübingen: Niemeyer Verlag.

Lüdeling, A. / Walter, M. (2010). Korpuslinguistik. In: H. J. Krumm / C. Chandrych / B. Hufeisen / C. Riemer (Hrsg.), Handbuch Deutsch als Fremd- und Zweitsprache (S. 315-322). BerlinNew York: de Gruyter.

Mel'čuk, I. (2001). Fraseologia y diccionario en la linguist. In: I. Uzcanga Vivar / E. L. Pombo / J. M. P. Velazco (Hrsg.), Presencia y renovación de la lingüística francesa (S. 261-310). Salamanca: Universidad de Salamanca.

Mukherjee, J. (2009). Anglistische Korpuslinguistik. Eine Einführung. Berlin: Erich Schmidt.

Polenz, P. von (1989). Funktionsverbgefüge im allgemeinen einsprachigen Wörterbuch. In: F. J. Hausmann, / O. Reichmann / H. E. Wiegand / L. Zgusta (Hrsg.), Worterbucher Dictionaries. Dictionnaires. Ein internationales Handbuch zur Lexikographie. An international encyclopedia of lexikograhy. Encyclopedie internationale de loxicographie (S. 882-887). Berlin-New York: de Gruyter.

Schmidt, V. (1968). Die Streckformen des deutschen Verbums. Substantivisch-verbale Wortverbindungen in publizistischen Texten der Jahre 1948-1967. Halle: Niemeyer.

Siepmann, D. (2009). Korpuslinguistik und Fremdsprachenunterricht. In: U. O. H. Jung (Hrsg.), Praktische Handreichung für Fremdsprachenlehrer (S. 321-330). Frankfurt am Main: Peter Lang.

Storrer, A. (2013). Variation im deutschen Wortschatz am Beispiel der Streckverbgefüge. In: Deutsche Akademie für Sprache und Dichtung / Union der deutschen Akademien der Wissenschaften (Hrsg.), Reichtum und Armut der deutschen Sprache (S. 171-209). Berlin: de Gruyter.

Taborek, J. (2011). Korpusbasierte Analyse der Phraseologismen. Dargestellt am Beispiel von ausgewählten verbalen Phraseologismen mit Komponenten aus dem Bereich Fußball. In: R. Lipczuk / M. Lisiecka-Czop / D. Misiek (Hrsg.), Phraseologismen in deutsch-polnischen und polnisch-deutschen Wörterbüchern. Theoretische und praktische Aspekte der Phraseologie und Lexikographie (S. 73-82). Hamburg: Dr. Kovač.

Taborek, J. (2017). Funktionsverbgefüge in bilingualen deutsch-polnischen Wörterbüchern. Korpusbasierte Analyse - syntagmatische Muster - Äquivalenz. In: V. Jesenšek / M. Enčeva (Hrsg.), Wörterbuchstrukturen zwischen Theorie und Praxis. H.t E.t Wiegand zum 80. 
Geburtstag gewidmet. Berlin: de Gruyter (= Lexikographica.Series Maior) (Pre-printVersion).

Wallner, F. (2013). Korpora im DaF-Unterricht - Potentiale und Perspektiven am Beispiel des DWDS. https://www.nebrija.com/revista-linguistica/korpora-im-daf-unterricht-potentiale-und-per spektiven-am-beispiel-des-dwds.html (Zugriff am: 5.08.2018).

Winhart, H. (2005). Funktionsverbgefüge im Deutschen. Zur Verbindung von Verben und Nominalisierungen. Tübingen. https://publikationen.uni-tuebingen.de/xmlui/handle/10900/46248 (Zugriff am: 5.08.2018).

Woźniak, J. (2016). Fachphraseologie am Beispiel der deutschen und der polnischen Fassung des Vertrags von Lissabon. Frankfurt am Main: Peter Lang.

Wörterbücher

PONS 2001 = Świrska, M. (Hrsg.) (2001). PONS. Duży słownik niemiecko-polski. Poznań: LektorKlett.

PONS 2007 = Dargacz, A. (Hrsg.) (2007). PONS. Wielki słownik niemiecko-polski. Poznań: LektorKlett.

PWN 2010 = Frączek, A. / Wiktorowicz, J. (Hrsg.) (2010). PWN. Wielki stownik niemiecko-polski. Warszawa: Wydawnictwo PWN.

Received: 10.08.2018; revised: 7.02.2019 